\title{
Leadership in the Setting of the Operating Room Surgical Team
}

\author{
David Arnold, MD, FACS ${ }^{1}$ James W. Fleshman, MD, FACS, FASCRS ${ }^{1}$ \\ ${ }^{1}$ Department of General Surgery, Baylor University Medical Center, \\ Dallas, Texas \\ Clin Colon Rectal Surg 2020;33:191-194.

\begin{abstract}
Address for correspondence James W. Fleshman, MD, FACS, FASCRS, Department of Surgery, Baylor University Medical Center, 3500 Gaston Avenue, 1st Floor, Roberts Hospital, Dallas, TX 75246 (e-mail: james.fleshman@bswhealth.org).
\end{abstract}

\author{
Abstract \\ Keywords \\ - transformational \\ leadership \\ - leadership styles \\ - coaching \\ - adapt
}

Leadership in the operating room requires the ability to adopt different styles under specific circumstances. Transformational leadership grows the team. The style used to guide the team can vary; coercive, visionary, affiliative, democratic, pacesetting, and coaching are all important styles in team leadership. The ability to adapt to different needs in the operating room by using the appropriate style is leadership.
The operating room has always been a place where an excellent outcome is expected. For an operating room to provide this on a consistent basis, there must be some understanding by the participants on how to make this happen. Certain rules and decorum are often handed down through the years or by the regulations that govern the way an operating room should work. Leadership in the operating room is essential to coordinate everyone's efforts for the good of the patient. Variations are often the norm. The leader of the surgical team in the operating room must be aware of these variables and constantly adapt. It is not always the loud, authoritative voice that commands the room. Leadership methods, styles, and behaviors are available to be taught as skills for the young surgeon. They should be a requisite in the training of anyone who is to one day lead a surgical team. ${ }^{1-4}$

The focus is often on the surgeon as the leader. A medical student that decides to enter a surgical residency has at least some inherent qualities of leadership. These qualities are often not enhanced through formal training during residency. Understanding the two types of leadership will allow a young surgeon to become more effective when it is the time and place to be the leader. Both transactional and transformational leadership methods are appropriate at certain times in the operating room.

Transactional leaders are often referred to as commanders. They employ a telling style of leadership. They motivate the followers through a system of rewards and punishments. There are two basic factors essential to transactional leadership: contingent reward and management by exception. Contingent reward provides motivation through rewards for effort and recognizes good performance. In the operating room, rewards and good performance are most often manifested as positive instead of negative feedback. Management-by-exception allows the leader to maintain the status quo. The leader using this method only intervenes when the subordinates do not meet expectations. This helps reduce the workload of the leader. Transactional leaders work within the system. They start solving challenges by fitting experiences into a known pattern. They want to know and use a step-by-step approach. They minimize variation within the organization. Transactional leadership works best in an established system, one where very little change is required. The team members know their roles and usually excel in those roles.

Transformational leadership focuses on maximizing the team's capability and capacity. Transformational leaders have four basic characteristics. The first is inspiration. They inspire the followers to find better ways of achieving a goal and are always focused on the greater good. The second is mobilization. They mobilize people into groups that can get work accomplished. Team members are matched up in ways to complement each other in achieving success. The next characteristic is morale. These leaders raise the well-being and motivation level of a group through excellent rapport. This forces the leader to be a part of the team, so that the team feels empowered and accountable in any outcome, positive or negative. Finally, they are excellent at conflict resolution.
Issue Theme Leadership; Guest Editor: James W. Fleshman, MD, FACS, FASCRS
Copyright (C) 2020 by Thieme Medical Publishers, Inc., 333 Seventh Avenue, New York, NY 10001, USA. Tel: +1(212) 760-0888.
DOI https://doi.org/ 10.1055/s-0040-1709442. ISSN 1531-0043. 
They allow their team to maximize their capability and capacity because friction between members is minimal. Transformational leaders are constantly working to change the system. They want to know what needs to be changed and then find ways to bring out that specific problem. Transformation leaders often solve challenges by finding experiences that show old patterns do not fit or work.

Transformational leaders work well in organizations where change is needed. They are excellent at communicating new ideas and having the team buy-in to those ideas. They excel at balancing short-term visions with long-term goals. These leaders often have integrity and high emotional intelligence which allows them to build rapport and increase the morale of the team. The ability to build strong coalitions and establish mutual trust is an essential trait of a transformational leader. On the converse, this type of leadership is ineffective in initial stages or ad hoc situations. It requires an existing structure so that change can be brought in as an improvement. It is also a bad fit in bureaucratic structures. They work best when each member of the team is on the same page.

It is therefore essential that a surgeon understand how both of these leadership styles work and in which instance each will work best. Situations arise in an operating room in which a surgeon must be able to change their style to provide the best outcome. The ability to understand and comprehend how to adapt will allow a surgeon and their team to provide a consistent outcome. It is therefore imperative to have some understanding of managerial and leadership styles. Knowing the fundamentals behind each style will allow the surgeon leader to have the proper tools at his or her disposal. It is possible then to choose when and where to make use of these fundamentals to make the operating room and surgical team more effective and efficient.

A directive or coercive style of management has the primary objective of immediate compliance from the entire team. It follows the mantra of "do it the way I tell you." There is close control over the entire team and uses threats and discipline as the primary means of motivation. This style is very effective in crisis situations. It also works well when deviations from the normal routine are risky. It is not effective when the components of the team are underdeveloped. It becomes very difficult to direct a part of the team to do something when they are incapable of performing that task. It is also not effective when the team is highly skilled. The team members will refuse to perform well because they do not require constant coercion.

The authoritative or visionary style has the primary objective of providing long-term direction and vision for the team. This is the "firm but fair" manager. The team is given clear direction but not in a coercive manner. The leader motivates by persuasion and provides feedback on task performance. This style of managing is most effective when clear directions and standards are needed. The leader that uses this style must be credible. The style fails when the leader is not credible because the team will be unable to follow the vision. It is not effective if the team is underdeveloped because they require more guidance.
The affiliative managerial style has the primary objective of creating harmony among the team and between the leader and the team, sometimes referred to as the "people first, task second" managerial style. This style motivates by trying to keep the team happy. It is most effective when combined with other managerial styles. It works well when routine tasks are the norm. This style demands only adequate performance from the team members, rather than excellence. When the team needs counseling or emotional support, it is useful. It is also an effective style at managing and resolving conflict. It is least effective during crisis situations as the task becomes more important than the people performing it. Affiliative management does not work if the baseline performance of the team is inadequate. It is difficult to keep the team happy and motivated when their work is subpar.

The participative or democratic style has the primary objective of building commitment and consensus. This is also known as the "everyone has input" style. The essence of this style is to motivate the team by rewarding effort. Everyone in the team works toward a common goal and is rewarded for that effort. This style is effective when the team works well together and has a stable work environment. It also requires the team to have experience and each member must be credible. The leader is then able to allow the team to be selfdirecting if the team is fully committed to the effort. It is least effective when the team needs to be coordinated. This style rarely works during a crisis, as the team tends to lose cohesiveness. If the team is not competent, the leader must provide too much close supervision for this style to be successful.

The pacesetting managerial style has the primary objective of accomplishing tasks to a high standard of excellence. This is the "do it myself" manager. The leader performs many tasks personally and expects the team members to follow through example. The leader motivates the team by setting high standards and expects self-direction from the members. This style is most effective when the team members are highly motivated and competent. They are more likely to follow the lead of the manager in this case. Little direction is required by the leader, as the team members are often experts. This style has difficulty when the workload requires assistance from individuals with less motivation and skill. It also tends to fall apart when the leader steps in too frequently and slows the progress, creativity, and innovation of the team.

The final managerial style to be discussed is coaching. It has the primary objective of long-term professional development of the team and its members. This is also known as the "developmental" manager. Coaching helps and encourages team members to develop their strengths, improve their performance, and achieve their ambition. The motivation for the team members is provided through opportunities for professional development. This style is effective when the members of the team have skills that need to be developed. The members of the team need to have a high level of self-motivation for this to work effectively. This style is effective during a crisis only if the members have adequate skill. If the leader lacks expertise, he or she is unable to draw out a high level of performance from the team. Members of the team must also have some similarity in the level of their performance or this style crumbles. 
The fundamentals of being a leader for the surgical team in the operating room are outlined. It should be useful to understand how they can be applied to various situations that arise in the operating room. It will also be beneficial to explore how a surgeon leader can impact the members of the surgical team on a continual basis. Knowing when to apply certain styles or methods will help create a cohesive, efficient, and trusting surgical team in a setting that can oftentimes be chaotic or unpredictable. The ability to incorporate different managerial styles into specific situations will allow a surgeon leader to get the very best out of the team. It is essential to understand when and how to use these styles and should become a mandatory part of the training of a surgical resident.

The patient and the type of procedure are fundamental to any operation. Patients encounter surgeons in varying environments. They may have an elective disease process that will require a simple outpatient procedure or a malignant process that will require hospitalization and ongoing treatment after radical surgery. They may present with an acute ailment that will necessitate urgent or emergency operation without much preparation or deliberation. These scenarios require the surgeon leader to use their skills and management styles to afford the patient the best outcome.

The patient expects the surgeon to be in control. The surgeon should make the patient feel at ease and comfortable with their unique surgical problem, instilling a sense of competency. By employing aspects of both affiliative and participative management styles, the surgeon allows the patient to feel both at ease and as if they are a part of the decision process. An empathetic surgeon will allow the patient to become comfortable with an otherwise uncomfortable or threatening event. The surgeon should give the patient time to ask questions and receive feedback which will relieve the patient's apprehension. The authoritative style now starts to morph into the equation as the surgeon gives a clear vision of what is expected to occur during and from the procedure. The patient now has a full understanding of what will happen and what is expected of them, so that they can participate fully in their care. If the scenario changes to a more urgent situation, then a more directive type style may be necessary. This will project a sense of control of the situation to the patient and personnel and bring calm yet clear objectives of what needs to be performed in specific time constraints.

The surgeon leader now turns to planning the actual procedure. The pacesetting and authoritative styles will be used most often now. Most of the planning and understanding of the task at hand need to be done by the surgeon alone. The patient and the operating room personnel need to have full confidence that the surgeon is capable of performing the intricacies of the specific procedure. The surgeon leader therefore needs to completely understand all the necessary components of the surgical procedure and display that they have received all educational requirements that go along with the surgery. Communicating with the surgical team prior to any planned procedure will set expectations with all the personnel and once again set a clear goal as to the objective. The surgical team can then use their own training and experiences to fulfill that vision. The surgeon leader should then provide appropriate feedback to the surgical team regarding their preparedness. This should be accomplished in a manner that will continue to provide the surgical team with motivation to perform at a high level for future surgical procedures.

The next step is the actual performance of the surgical procedure. Any elective procedure requires coordination of the surgical team. This may require the surgeon leader to be a more participative leader. The correct equipment and environment need to be provided. This often occurs without much input from the surgeon. He or she needs to allow the team to participate and give input when needed and build a commitment to the process.

Once the proper equipment and supplies are ready, the surgeon becomes more of an authoritative leader again. The surgeon should take an active role in a time-out process to ensure that the correct procedure and site are being performed. The surgical scrub expects the surgeon to ask for specific instruments throughout the procedure. Feedback from the surgeon to the surgical scrub will enhance this aspect and should be encouraged. The circulating nurse will also provide essential supplies throughout the procedure and should be motivated by feedback from the surgeon. If a situation arises that is unexpected, then the team and leader need to show adaptability and flexibility. The scrub and circulator need to know that their input in these circumstances can often lead to solutions to these unexpected events. The surgeon leader needs to allow the operating room staff to be engaged in these decisions as they do not always need guidance when they may have prior experience and expertise that can help.

In an emergency or trauma situation, the planning and preparation are not always possible. The necessary equipment and supplies are not always readily available. The expected flow of a procedure is often quite variable, and the surgeon leader needs to rely heavily on a combination of directive and participative managerial styles. There needs to be clear directions and immediate compliance from the operating room personnel, but the surgeon must also create an atmosphere where the entire team can perform their tasks with their specific talents. Resilience by the surgical team is paramount as they can sometimes be stretched to the limit by the stressors of the situation. The team should know their capabilities and have the awareness that their skills and attitudes will influence the outcome of the procedure. The reward of a job well done in the face of trying circumstances, no matter what the eventual outcome, will provide a competent team with motivation for future situations.

Once the surgical procedure is complete, a debriefing process will prove useful. The surgeon leader can provide real-time feedback in a controlled, calm manner, which may not be accomplished during procedure. The surgeon should learn to develop interpersonal skills that will make this a learning process for the entire team. Positive feedback and constructive criticism are both appropriate during the debriefing but need to be delivered with insight and thought to the audience. Selflessness from the surgeon is also a requirement, 
as the team needs to provide feedback as well. It is not always an easy thing for a surgeon to do, but listening to the operating room team can only augment future collaborations and allow the team to improve further. Whether the need is better communication, interpersonal interaction, or behavior, the debriefing process will certainly provide for ongoing education for the entire team.

A unique situation for many surgeon leaders is the opportunity to instruct in the operating room. Many surgeons take part in residency programs. The goal of training another individual to perform surgery and to take appropriate care of patients takes many leadership styles and traits. The surgeon leader needs to be able to train using the directive style in many instances as their directions need to be followed with immediacy. The young trainee needs to be closely controlled at times. This will allow the resident to learn in the setting of the operating room where control is essential. Early on in training, the resident will respond well to a pacesetting style as they can learn to emulate their surgical teachers. They will learn from the example set before them. The authoritative style imparts a clear understanding of the learning objectives and should be employed early on in training as well. As the resident gains more experience, both the affiliative and participative styles come in to play. The resident needs to feel like they are making decisions, especially in the operating room. This will make them feel like they are an important part of the team. They will also start to gain the respect of the operating room personnel as they begin to show that they are making decisions and performing the necessary steps of the procedure. The resident now can become motivated by being part of the team and then growing into the leader of the team.

The overriding style of a surgeon teacher should be one of coaching. The surgeon teacher is the quintessential developmental manager. They need to understand when to provide supervised experiences for the resident so that they can develop the necessary skills. The surgeon leader/teacher also needs to provide opportunities for the resident to use their skills and education on their own. Only in this manner can a surgical trainee develop into a surgical leader. They will learn to lean on their strengths and their experiences, so that they too can be the leader of a team in the operating room.
Surgical leadership in the operating room requires an understanding of many qualities. The surgeon leader needs to learn empathy and emotional intelligence. Recognizing the impact of one's own behavior on others' emotional and cognitive state is the key to managing one's own self. Leading requires an understanding of the skills and attitudes needed for that role. Only by looking inward and constantly evaluating how one practices leadership, can a surgeon truly learn the art of leadership. They need to understand the entire process of developing a team in the operating room. The principle of treating others as you want to be treated when interacting with each individual and the ways that these interactions can influence the outcome of a surgical procedure, are skills that should be learned.

A thorough comprehension of managerial styles and how to implement them in each operating room scenario is paramount to blossoming into an effective leader. The surgical leader needs to have insight into when and where each style will afford the best result. They should develop the ability to change from one style to another depending on the situation. By being flexible and adaptable with their leadership qualities, a surgeon can take an operating room team to peak performance. Even so, the surgeon leader is not the most important person in the operating room. The measurement of success should always be the outcome of the patient. The most important person in the operating room should always be the patient.

Conflict of Interest

None declared.

\section{References}

1 Goleman D. Leadership that gets results. In: Harvard Business Review. 2016

2 Constantino T. Which of the 6 leadership styles defines you? In: Leadership. 2016

3 Cardinal R. 6 Management styles and when best to use them - the leaders tool kit. In: Leaders in Heels. 2013

4 Spahr P. What is transformational leadership? How new ideas produce impressive results. In: Leadership is Learned. 2014 